\title{
Deriving topological relations from topologically augmented direction relation matrices
}

\author{
Matthew P. Dube \\ Department of Computer Information Systems, University of Maine at Augusta, USA
}

Received: December 18, 2019; returned: July 16, 2020; revised: July 23, 2021; accepted: August 23, 2021.

\begin{abstract}
Topological relations and direction relations represent two pieces of the qualitative spatial reasoning triumvirate. Researchers have previously attempted to use the direction relation matrix to derive a topological relation, finding that no single direction relation matrix can isolate a particular topological relation. In this paper, the technique of topological augmentation is applied to the same problem, identifying a unique topological relation in $28.6 \%$ of all topologically augmented direction relation matrices, and furthermore achieving a reduction in a further $40.4 \%$ of topologically augmented direction relation matrices when compared to their vanilla direction relation matrix counterpart.
\end{abstract}

Keywords: qualitative spatial reasoning, interoperability, direction relations, topological relations, topological augmentation

\section{Introduction}

In the seminal work of qualitative spatial reasoning, Kuipers [25] declared that three particular types of classifications can be applied to qualitative spatial vocabulary:

- topology, the set of properties of a collection of objects that do not change under rubber sheet geometric approaches (including containment and connection) [16,39],

- direction, the set of properties that provide a concept of orientation between two or more objects (including egocentric and allocentric views) [20,21,38], and

- distance, the set of properties that dictate how far two objects are from one another (including such concepts as near and far) [47]. 
Over four decades of research has provided a litany of representations for each of these types of qualitative descriptors, most notably topology $[12,15-17,22,26,28,29,34,37,39$, $43,44,46]$ and direction $[4,20,21,32,35,36,38]$. While the set of formalizations has grown drastically in both of these instances, there are relatively few instances where topology and direction are treated together $[8,24,42]$.

Since direction and topology are intricately entwined together [18], it is essential to consider how direction relations can be transformed into topological relations and vice versa. While topological information does not provide much in the way of opportunities for informing knowledge of direction, the reverse is not true: direction provides context for topological relations, particularly when considering qualitative spatial partitions that represent a directional view of space, such as the direction relation matrix [21] or the objects-interaction matrix [4]. Guo and Du [23] have studied the transferability of direction relations into topological relations in the embedding space of the direction relation matrix [21], demonstrating that no single direction relation matrix is capable of isolating a specific topological relation, at best limiting the possibilities to two between a pair of simple regions.

Dube [8] proposed a spatial formalism to directly pair topological and directional information called topological augmentation. In this formalism, the binary classification of intersection from the direction relation matrix [21] is replaced with a topological spatial relation [17] between the embedding tiles derived by the ground object and the figure object itself, thus conveying knowledge of the intersection of boundaries. This simple change produces an inflation from 218 realizable direction relation matrices to 3,048 realizable topologically augmented direction relation matrices.

This paper explores to what extent the additional information provided by topological augmentation facilitates the interchange of directional and topological information for two simple regions in $\mathbb{R}^{2}$. The remainder of the paper proceeds as follows: Section 2 details the three central spatial formalisms used in this paper: the 9-intersection [17], the direction relation matrix [21], and topological augmentation [8]. Section 3 details the work of Guo and $\mathrm{Du}$ [23] to determine how direction relation matrices map to topological relations. Section 4 presents a theorematic account of both availability of topological relations and the discernment of topological relations through topological augmentation. Section 5 provides an analysis of the output of these theorems, demonstrating the gains of topological augmentation. Section 6 provides conclusions and calls for future work.

\section{Spatial formalisms}

This paper is based off a foundation of three formalisms: the 9-intersection [17], the direction relation matrix [21], and topological augmentation [8]. The next three subsections detail these three formalisms as they pertain to this paper. In all cases, we will consider two objects: a figure object $A$ and ground object $B$ [45].

\subsection{9-intersection matrix}

The 9-intersection matrix is a qualitative spatial reasoning formalism that is founded upon three important interrelated topological concepts: interior, boundary, and exterior [17]. To provide context, these terms are defined as follows [1]:

WWw.josis.org 
Definition 2.1. Let $X$ be a subset of an embedding space $S$ with a defined topology $T$. The interior of $X$, denoted as $X^{0}$, is the largest open set $O \subseteq X$.

Definition 2.2. Let $X$ be a subset of an embedding space $S$ with a defined topology T. The closure of $X$, denoted as $\bar{X}$ is the smallest closed set $C$ such that $X \subseteq C$.

Definition 2.3. Let $X$ be subset of an embedding space $S$ with a defined topology $T$. The boundary of $X$, denoted as $\partial X$, is the set $B=\bar{X} \backslash X$.

Definition 2.4. Let $X$ be a subset of an embedding space $S$ with a defined topology $T$. The exterior of $X$, denoted as $X^{-}$, is the set $E=S \backslash X^{-}$.

Definitions 2.1-2.4 reflect the standard vocabulary that is used to define the 9intersection. The 9-intersection matrix records the interplay between the interior, boundary, and exterior of the figure object $A$ with the interior, boundary, and exterior of ground object $B$. All of its elements are declared empty or non-empty. This formalism can be applied to a wide variety of topological spaces and sets within them. Researchers have studied numerous types of relation sets with this method $[7,10,11,14,19,30,33,40,41]$, however the most well-known of these sets is the set of relations that pertain to the class of simple regionregion relations, as shown in Figure 1 [17]. This set of relations has a defined composition table that is strong $[13,31]$. The composition table is shown as Figure 2.
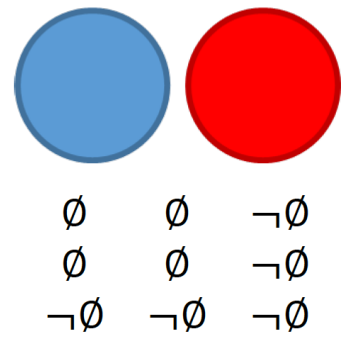

disjoint (d)

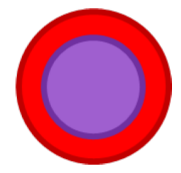

$$
\begin{array}{ccc}
\neg \emptyset & \emptyset & \emptyset \\
\neg \varnothing & \varnothing & \varnothing \\
\neg \varnothing & \neg \emptyset & \neg \emptyset \\
\multicolumn{4}{c}{\text { inside (i) }}
\end{array}
$$

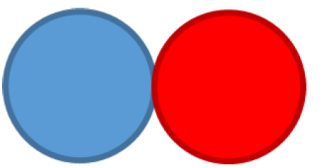

$\varnothing \quad \varnothing \quad \neg \varnothing$

$\varnothing \quad \neg \varnothing \quad \neg \varnothing$

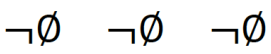

meet $(m)$
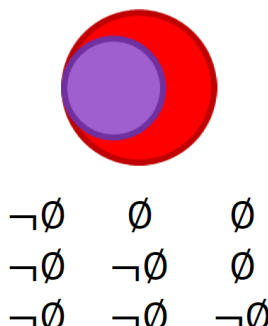

$\neg \emptyset \quad \neg \emptyset \quad \neg \emptyset$

coveredBy $(c B)$
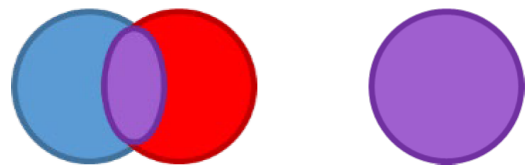

$\begin{array}{cccccc}\neg \emptyset & \neg \emptyset & \neg \emptyset & \neg \emptyset & \emptyset & \emptyset \\ \neg \emptyset & \neg \emptyset & \neg \emptyset & \emptyset & \neg \emptyset & \emptyset \\ \neg \emptyset & \neg \emptyset & \neg \emptyset & \emptyset & \emptyset & \neg \emptyset\end{array}$

overlap (o)

equal (e)
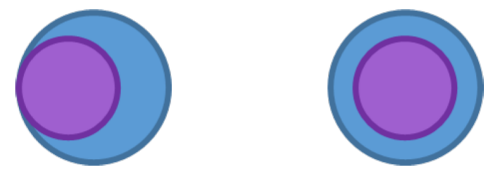

$$
\begin{array}{cccccc}
\neg \emptyset & \neg \emptyset & \neg \emptyset & \neg \emptyset & \neg \emptyset & \neg \emptyset \\
\varnothing & \neg \emptyset & \neg \emptyset & \emptyset & \emptyset & \neg \emptyset \\
\varnothing & \varnothing & \neg \emptyset & \emptyset & \emptyset & \neg \emptyset \\
\multicolumn{4}{c}{\text { covers (cv) }} & \multicolumn{3}{c}{\text { contains (ct) }}
\end{array}
$$

Figure 1: The eight simple region-region relations identified by the 9-intersection [17]. 


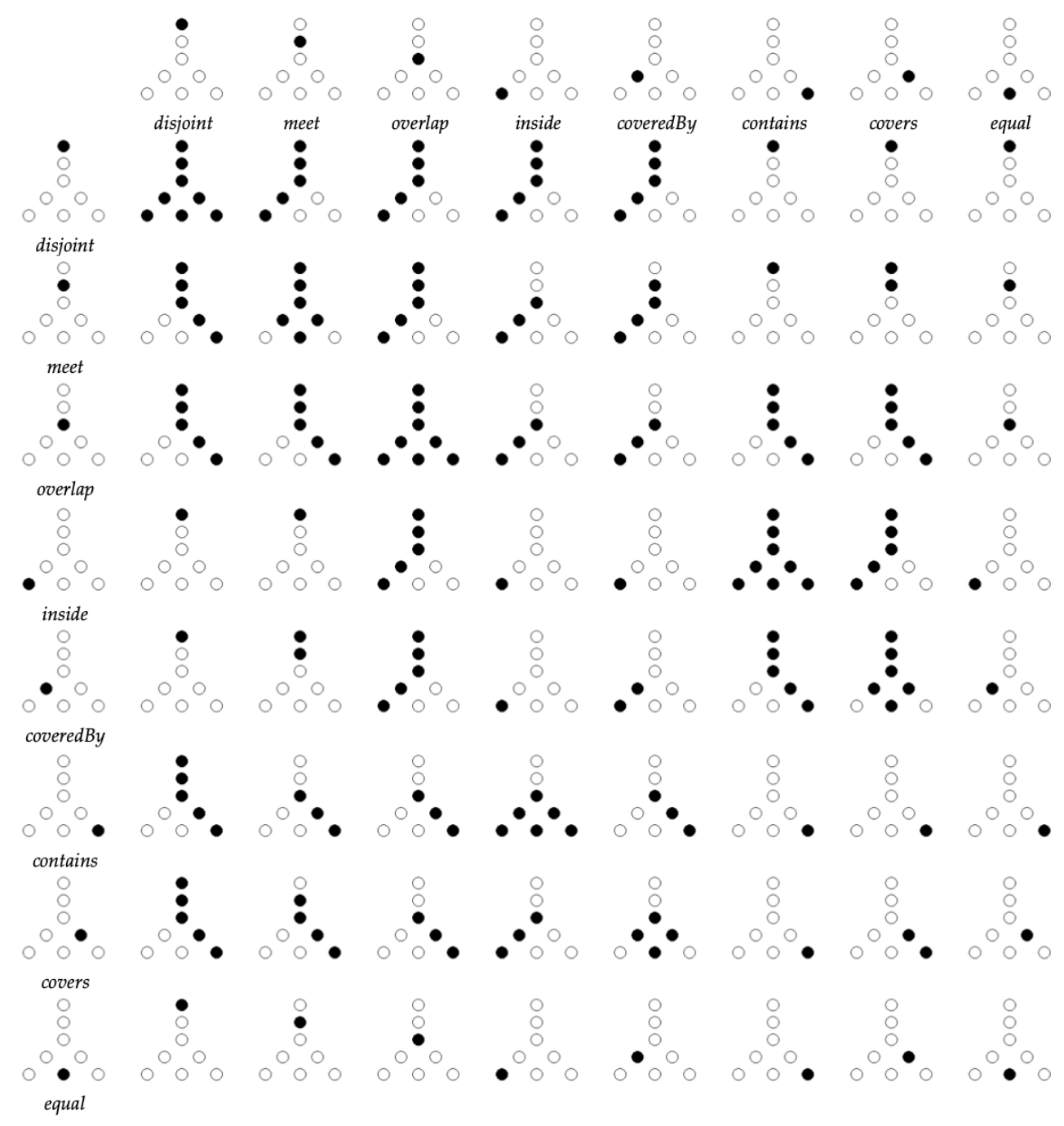

Figure 2: The composition table of the eight simple region-region relations [13].

\subsection{Direction relation matrix}

The direction relation matrix is a formalism that is constructed to detail the direction between a figure object $A$ and a ground object $B$ [21]. It accomplishes this goal by subdividing a space by constructing a minimum bounding rectangle (MBR) around the object $B$, extending the lines of the MBR to span the space, and then qualitatively intersecting A with the subdivisions, called tiles, formed by the subdivision of the space by the MBR and its extension, as shown in Figure 3. This approach allows for the structure of the formalism 
to resemble the 9-intersection matrix. Barrett [2] applied a similar concept to a more arbitrary space defined from the perspective of an airplane pilot. Figure 4 demonstrates which direction relation matrix symbols are viable for two simple regions.

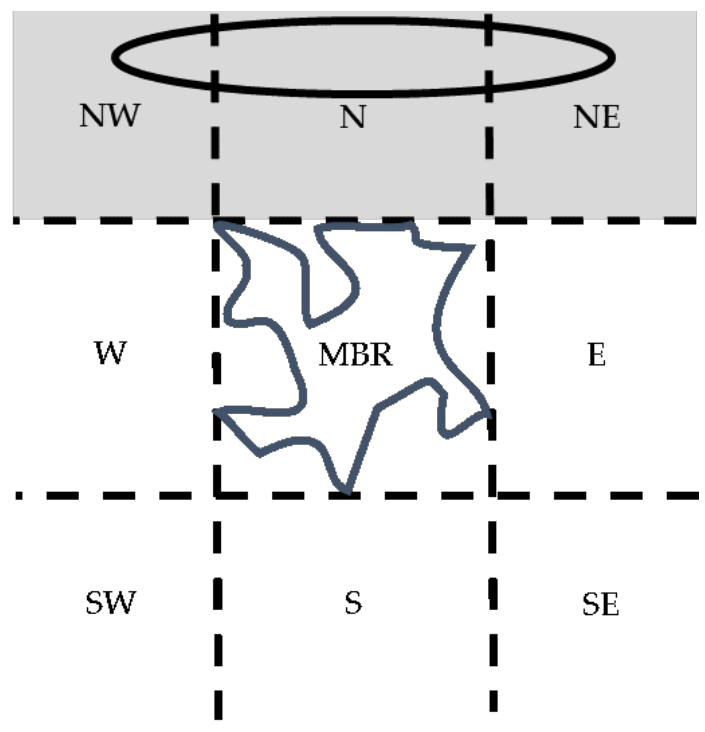

Figure 3: An example of two objects $A$ and $B$, such that the MBR of $B$ generates a partition of space, and $A$ qualitatively intersects it, forming its direction relation matrix.

Section 3 will assess the usage of the direction relation matrix as a mechanism for the identification of a topological relation between the two objects defining the symbol [23]. Section 2.3 will detail a recent innovation to the direction relation matrix: topological augmentation [8].

\subsection{Topological augmentation}

Topological augmentation [8] is the marriage of the 9-intersection [17] to the direction relation matrix [21]. While the direction relation matrix focuses on a binary intersection [21], topological augmentation assigns a qualitative topological relation to each tile in the space reflecting the relationship between figure object $A$ and that tile. While the direction relation matrix misses boundary contact, topological augmentation provides this information in a qualitative way. Figure 5 demonstrates an application of topological augmentation.

To efficiently detail the results of topological augmentation, Dube [8] detailed a set of congruence classes that can be identified between direction relation matrices. This set of congruence classes is presented as Figure 6.

Not all topological relations can be applied to any cell in topological augmentation. The reason for this is two-fold: (1) the tiles $N_{B}, S_{B}, E_{B}, W_{B}, N E_{B}, N W_{B}, S E_{B}$, and $S W_{B}$ all have indefinite extents to account for the infinite expanse of $\mathbb{R}^{2}$ in all directions, and (2) the direction relation matrix and its binary intersections present a set of constraints that define the topological relations available. While the full set of constraints defining topo- 


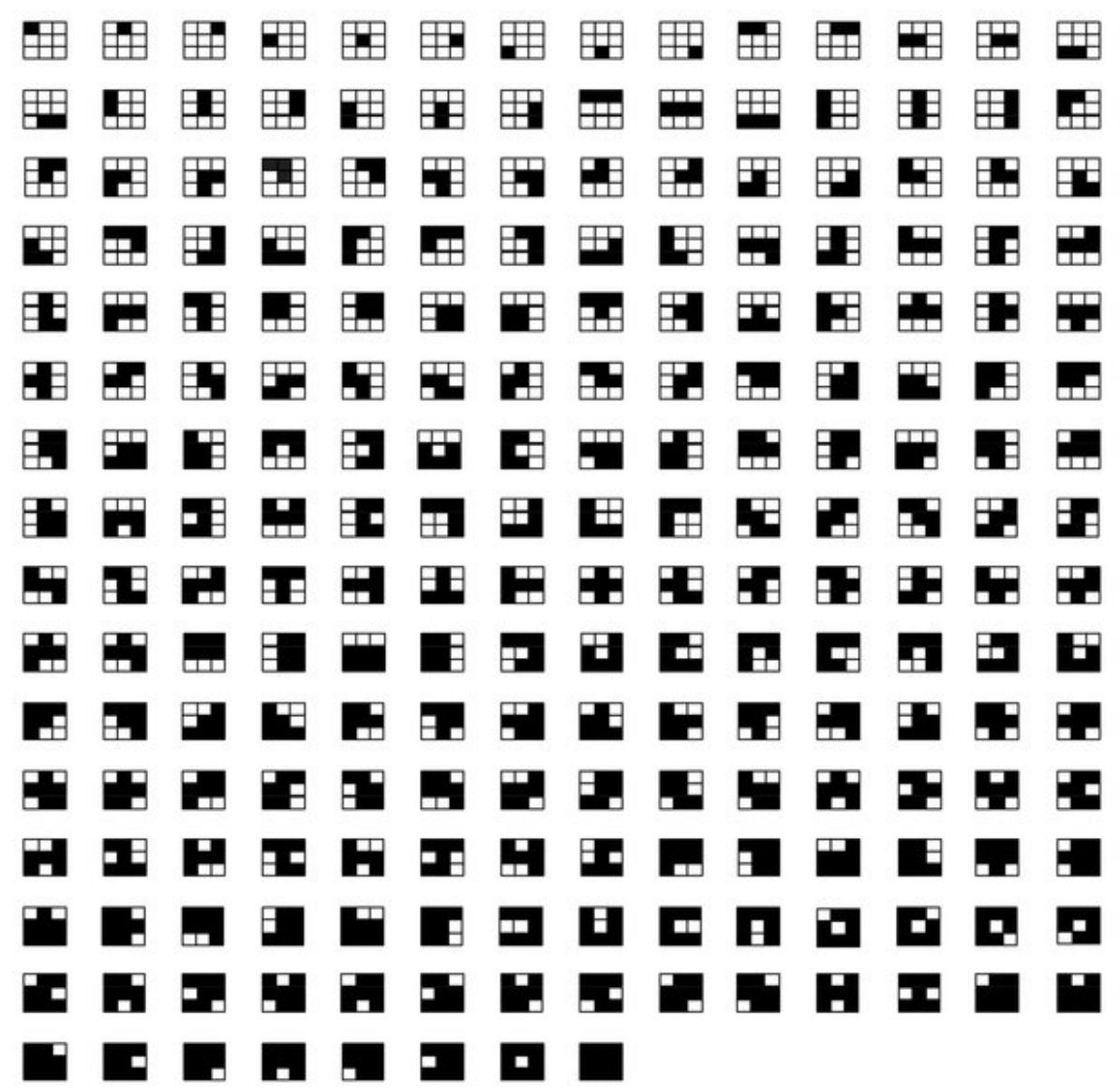

Figure 4: The available set of direction relation matrix symbols for two simple regions [21].

logical augmentation is not necessary for this paper (as it will assume valid topologically augmented direction relation matrix symbols), the most basic of the constraints are [8]:

- If a tile has an empty intersection, the topological relation between the figure object $A$ and it must be either disjoint or meet,

- If a tile has a non-empty intersection, the topological relation between the figure object $A$ and it must be any of overlap, equal, inside, coveredBy, contains, or covers, and

- If a tile is unbounded (as in the first rationale for restrictions to possible topological augmentation), figure object $A$ may only have topological relation disjoint, meet, overlap, inside, or coveredBy to that tile, whereas figure object $A$ may have any topological relation with a bounded tile.

All other constraints to viable topologically augmented direction relation matrix symbols are constraints that apply across specific combinations and configurations of empty and non-empty tiles as prescribed by the viable direction relation matrices [8].

www.josis.org 

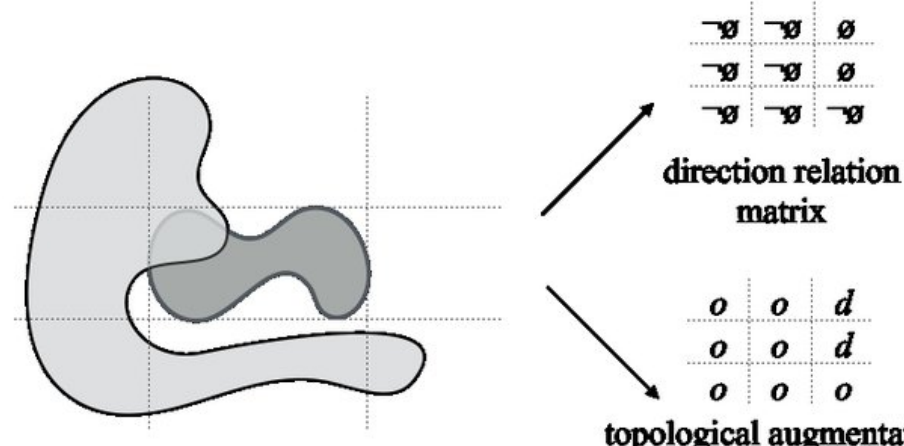

\section{topological augmentation \\ on the direction relation \\ matrix}

Figure 5: Topological augmentation applied to a pair of objects. The corresponding direction relation matrix symbol is also shown [8].

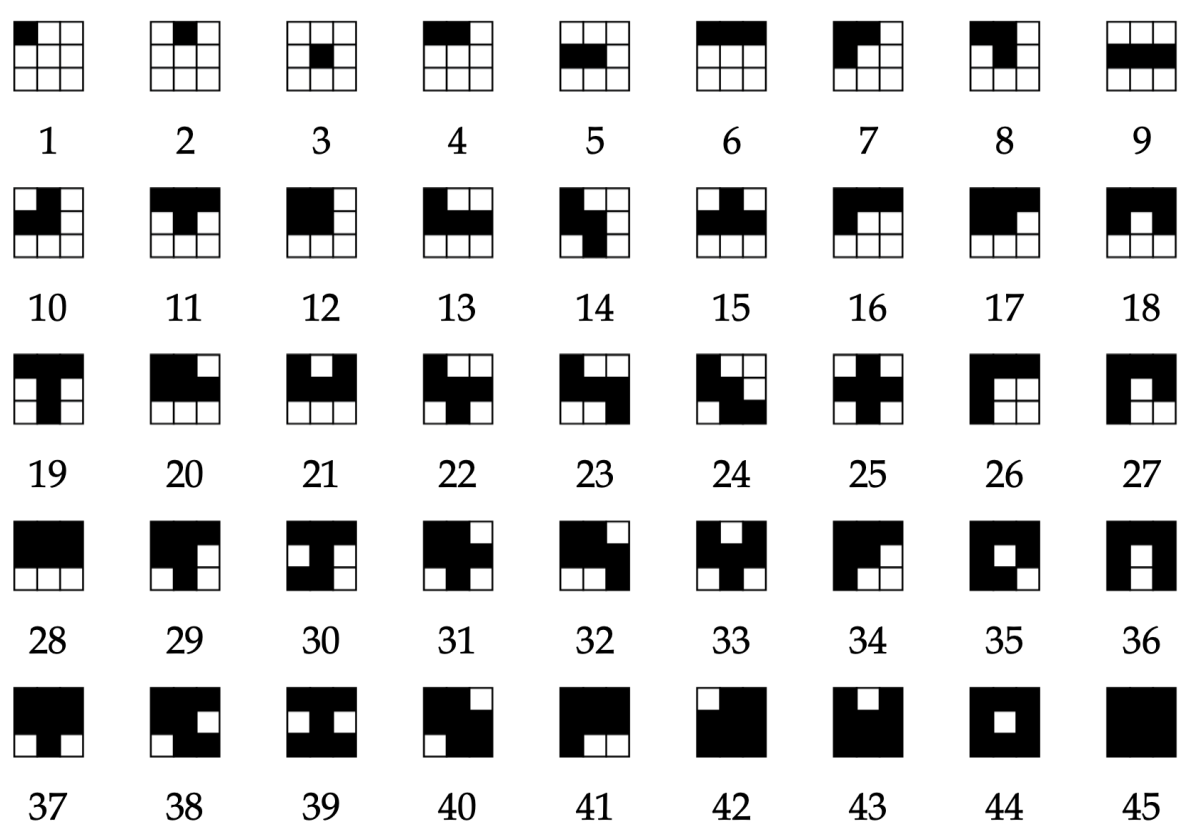

Figure 6: Congruence classes of direction relation matrices [8].

Section 4 will apply topological augmentation to the problem of converting a direction relation matrix to a topological relation. 


\section{Determining a topological relation through the direction relation matrix}

Guo and $\mathrm{Du}$ [23] conducted a study motivated by spatio-temporal data mining to attempt to use the direction relation matrix as a means for determining the available topological relation between the regions defining the direction relation matrix symbol. This process is governed by a set of rules that are proven in their work, and presented here. Each proof (not presented) is directly supported by the composition table of 9-intersection relations between two simple regions [13].

In each of the forthcoming rules, $R_{A B}$ refers to the non-empty intersections between object $A$ and the direction tiles. For example, Figure 3 shows $R_{A B}=\left\{N W_{B}, N_{B}, N E_{B}\right\}$, represented in row 2, column 8 of Figure 4, and in congruence class 6 of Figure 5.

Rule 3.1. If $R_{A B}$ represents the direction relation between $A$ and $B$, and $M B R(B) \notin R_{A B}$, the topological relation between $A$ and $B$ must be either disjoint or meet.

Rule 3.2. If $R_{A B}$ represents the direction relation between $A$ and $B$, and $R_{A B}=M B R(B)$, then the topological relation between $A$ and $B$ can be any of disjoint, meet, overlap, equal, inside, coveredBy, or covers.

Rule 3.3. If $R_{A B}$ is the direction relation between $A$ and $B, M B R(B) \in R_{A B}$, and $R_{A B}$ includes at most three out of $N_{B}, S_{B}, E_{B}$, and $W_{B}$, the possible topological relations between $A$ and $B$ are disjoint, meet, overlap, and covers.

Rule 3.4. If $R_{A B}$ is the direction relation between $A$ and $B$, and $\left\{M B R(B), N_{B}, S_{B}, E_{B}, W_{B}\right\} \subseteq$ $R_{A B}$, then the possible topological relations between $A$ and $B$ are disjoint, meet, overlap, covers, and contains.

By applying these rules to the congruence classes from Figure 6, the set of topological relations possible for a given direction relation matrix $R_{A B}$ can be inventoried at the congruence class level, as shown in Figure 7. Any element of the same congruence class may produce the same topological relations as any other element of that congruence class.

The substantive issue with the direction relation matrix is that the omission of boundary information prevents many topological relations from being distinguished from one another. In fact, as shown in Figure 7, no direction relation matrix is capable of isolating a single topological relation. While it can limit the options available in all cases, no identifying conditions are present. Figure 8 provides an example of this problem (from congruence class 4$)$.

An additional problem with the method of Guo and Du [23] is exposed within Rule 3.3. Having at most three elements of $N_{B}, S_{B}, E_{B}$, and $W_{B}$ does induce the relation covers between the collection of tiles and the ground object $B$, this does not guarantee that all possible cases are capable of producing all four of disjoint, meet, overlap, or covers. An easy example of this is seen in $R_{A B}=\left\{W_{B}, M B R_{B}, E_{B}\right\}$, the subject of Theorem 3.1.

Theorem 3.1. If $R_{A B}$ includes $M B R(B), E_{B}$, and $W_{B}$ without containing $N_{B}$ or $S_{B}$, then overlap and covers are the only topological relationships possible between $A$ and $B$. The same is true for the orthogonal directional case.

Proof. Consider a hypothetical scenario where $E_{B}$ and $W_{B}$ are contained in $R_{A B}$, but not $N_{B}$ or $S_{B}$ (such as Figure 9). Without loss of generality, consider a non-self-intersecting

www.josis.org 


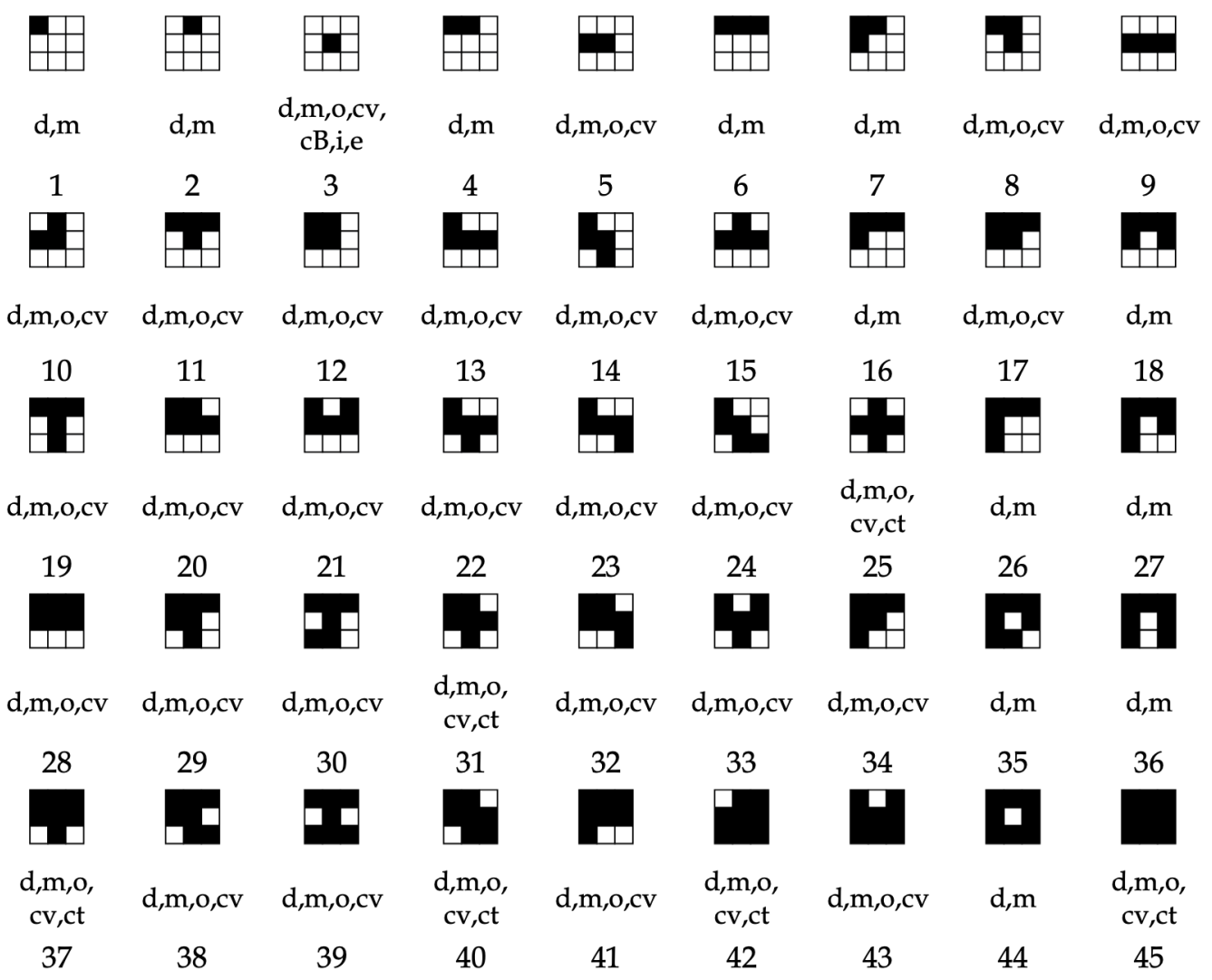

Figure 7: Rules from Guo and Du [23] applied to congruence classes.

curve connecting the boundaries of $E_{B}$ and $W_{B}$ that does not leave the MBR. By definition of the MBR, that line must impact the boundary of object $B$. If $B=M B R(B)$, then disjoint and meet are not possible as there is no way to connect $A$ to the opposite side of the MBR without intersecting the object $B$. Otherwise, consider $M B R(B)$ as an embedding space, including its boundary. $B$ partitions this space into multiple components, with only one of them $(B)$ allowed to have a 1-meet relationship with the other partitions. For disjoint or meet to be possible, $A$ cannot intersect $B$, thus it must go through a point connecting the exterior partitions with each other or to a non-empty tile, but thinning $A$ to a single point will subdivide $A^{0}$ into multiple parts to facilitate crossing over the MBR without intersecting $B^{0}$. This is a contradiction to the definition of simple region.

This assertion impacts several of the congruence classes from Figure 7. Classes 9, 13, 19, $21,23,30$, and 39 thus have an immediate reduction in their available reductions, yet this does not allow for unique identification. The updated congruence class designations are shown in Figure 10. 


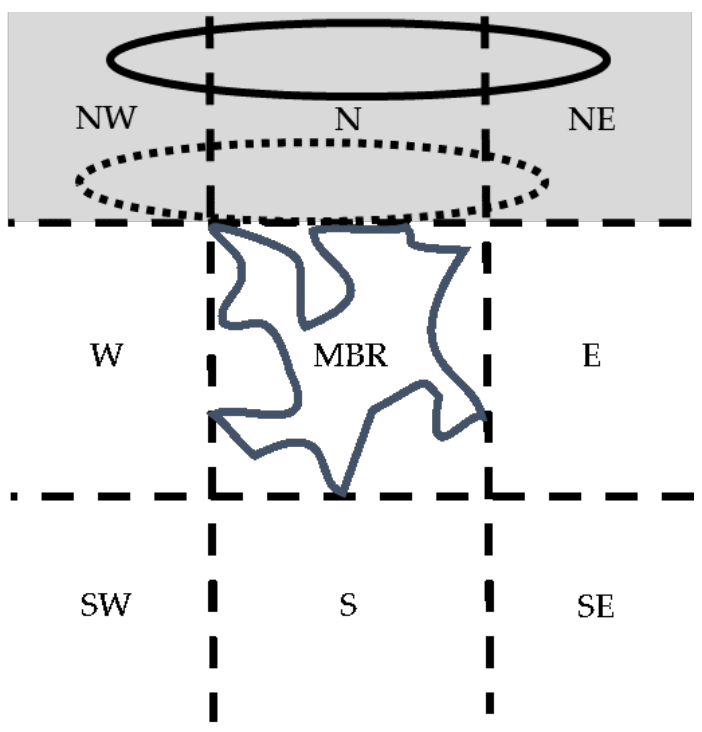

Figure 8: An example of two objects producing the same direction relation matrix that have two different topological relations: disjoint (solid) or meet (dotted).
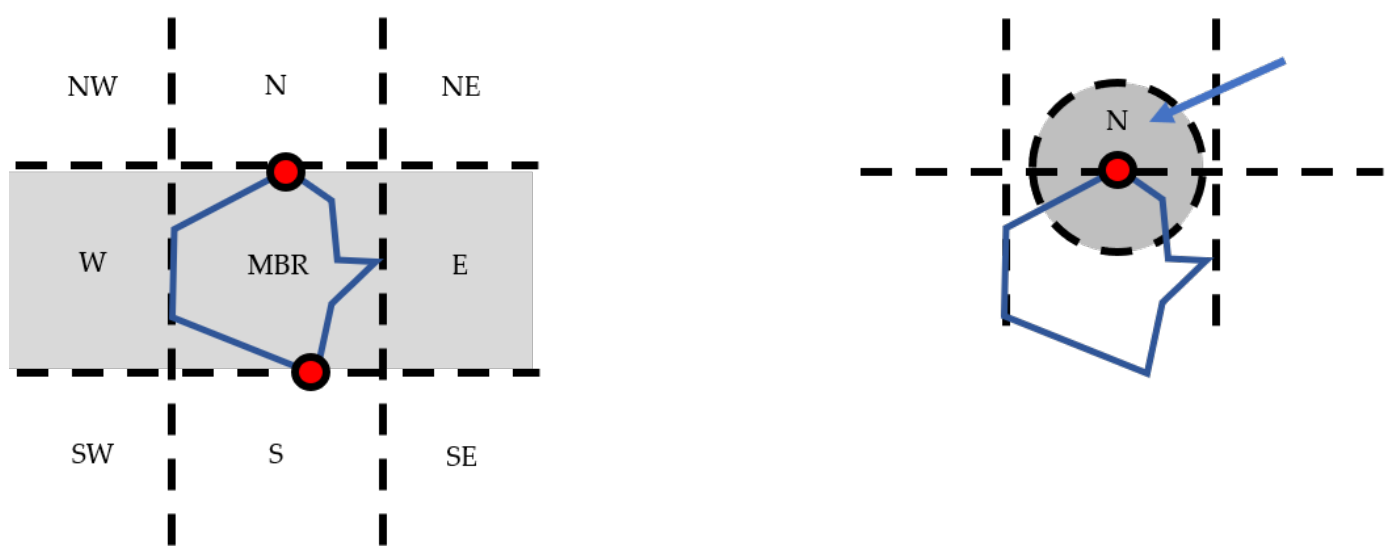

Figure 9: Example used for Theorem 3.1. For the object $A$ to meet object $B$, object $A$ (with non-zero thickness as it is a simple region) is forced to pass through the common point between $\operatorname{MBR}(B), B$, and $N_{B}$ without intersecting either $B$ or $N_{B}$. The same is true for the corresponding southern extremity.

\section{Applying topological augmentation to the topological de- termination problem}

Since topological relations are ultimately a foundational backbone of our languages $[9,18,27]$, the inability of the direction relation matrix to transfer to any unique topolog-

wWw.josis.org 


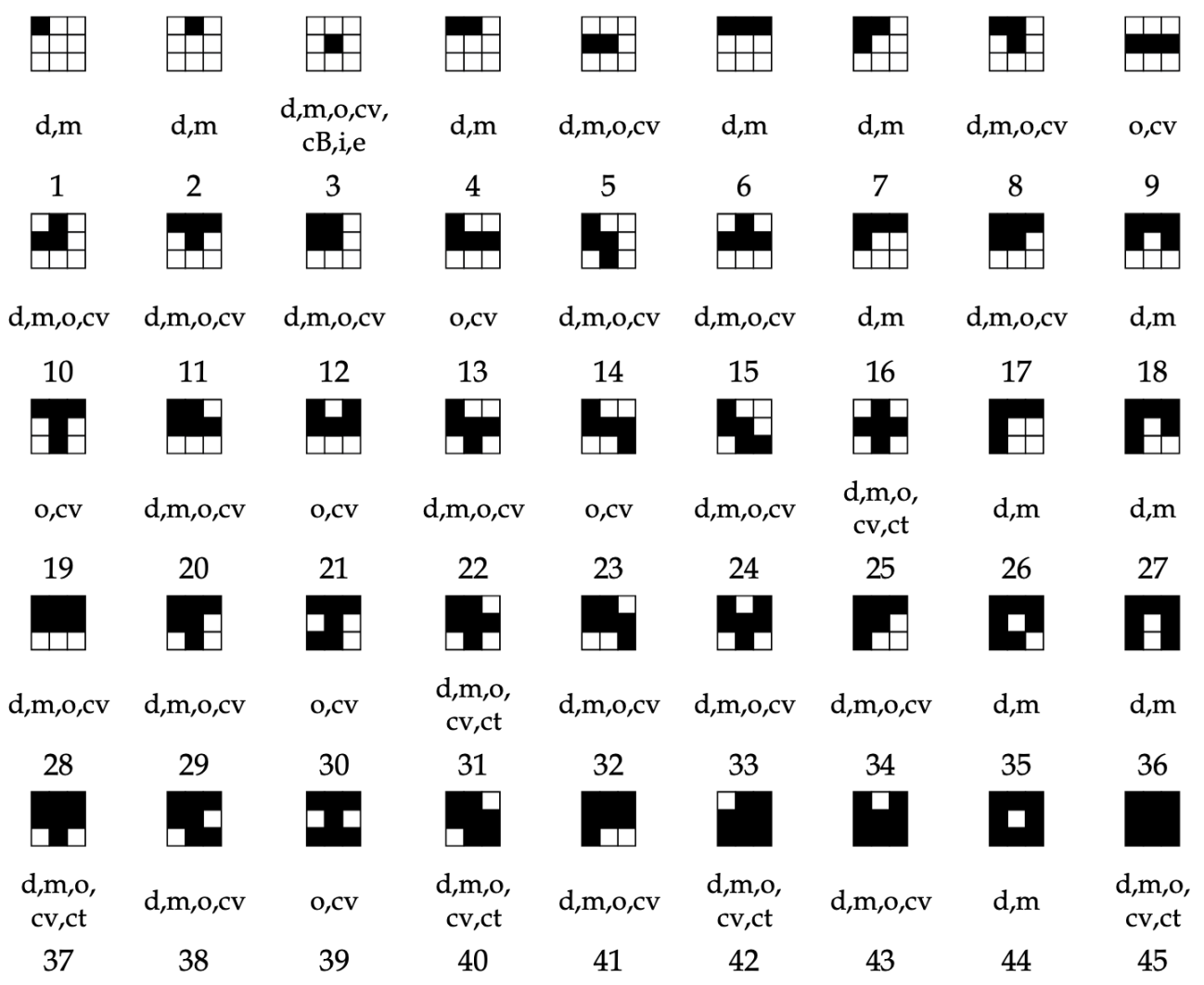

Figure 10: Rules from Guo and Du [23] applied to congruence classes, adjusted as a result of Theorem 3.1.

ical relation is a major issue. Topological augmentation can be applied to this problem, something that has at least an opportunity to succeed in topological identification due to maintaining boundary contact within the formalism.

Similar to Guo and Du [23], topological augmentation defines a set of rules that govern which topological relations can result from a topologically augmented direction relation matrix symbol. Topological augmentation's rules, however, differ insofar as it has a larger diversity of symbols from which to work. The following rules can be established using the terms of topological augmentation.

Rule 4.1. The relation equal is only possible if the relation to the $M B R$ is coveredBy or equal and at least all of $N_{B}, S_{B}, E_{B}$, and $W_{B}$ are meet.

Proof. The relation equal requires both $A$ and $B$ to be the same. The coveredBy or equal assertion is the exact relation between an MBR and the object that defined it [37]. Since $A$ must be restricted to the minimum bounding rectangle in this case and must maintain boundary contact with all four edges of the MBR, by default, it must then hold the relationship meet 
with each of the four listed tiles, as the MBR also holds the relation meet with these four tiles as well.

Rule 4.2. The relation inside is only possible if all neighbors of the MBR have relation disjoint and the relation to the $M B R$ tile is also inside.

Proof. The relation inside between $A$ and $B$ can be determined specifically through composition of $\operatorname{rel}(A, B)$ to $\mathrm{rel}(B, \operatorname{MBR}(B))$ to $\mathrm{rel}(\mathrm{MBR}(B)$, tiles $)$. inside; $($ coveredBy or equal $)=$ inside and inside $;$ meet $=$ disjoint .

Rule 4.3. The relation coveredBy is only possible if all neighbors of the $M B R$ have relation disjoint or meet.

Proof. Like 4.2, this can be asserted through composition. Since coveredBy allows for boundary contact between $A$ and $B, A$ can thus intersect the boundary of the MBR, and thus the boundary of any neighboring tile. (inside or coveredBy); $($ coveredBy or equal $)=($ coveredBy or inside) and (coveredBy or inside); meet $=$ (disjoint or meet $)$.

Rule 4.4. The relation contains is only possible if all four edge neighbors of the MBR are overlap.

Proof. This can be demonstrated through composition as well. The relation between $A$ and the MBR is contains ; $($ coveredBy or equal $)=($ contains, covers, overlap $)$. If the relationship between $A$ and the MBR is contains, then all neighbors of the MBR must have relation overlap as contains; meet $=($ contains, covers, overlap $)$, but covers and contains are not possible between $A$ and a partially bound area. If the relation is covers, then the possibilities are (meet, overlap, covers, contains). We have already demonstrated that covers and contains are not possible. The instance of meet in this resulting answer space can only exist for the ordinal direction tiles, as $A$ contains $B$ forces the $A$ to extend beyond the MBR in every place where $B$ contacts the MBR, which must extend into the cardinal directions. If the relation is overlap, the possibility of disjoint is added, which is no different than the exclusion rule for meet.

Rule 4.5. The relation covers is only possible if all four edge neighbors individually are not disjoint with respect to $A$.

Proof. Like Rule 4.4, the worst case scenario for this rule is the case when $A$ is the MBR. In this case, $A$ has defined relation meet with any of the other tiles. Any extension of $A$ such that it maintains boundary contact with $B$ will still satisfy covers, even if it moves the four edge neighbors beyond meet in the conceptual neighbourhood graph in the direction of overlap.

Rule 4.6. The relation overlap is only possible if the $M B R$ has relation overlap, coveredBy, or inside.

Proof. The relation overlap dictates that the object $A$ must share points with the object $B$, but both $A$ and $B$ must have points that remain separate from one another. If $A$ inside MBR, then $B$ automatically has points that are not part of $A$, as $B$ must contact the boundary of its MBR. Unless $B$ is equal to the MBR, it is trivially possible for $A$ to extend in some direction beyond $B$, thus allowing it to have points not in common with $B$, yet still in the MBR. By the same token, if $A$ coveredBy MBR, then $A$ has room to grow within the MBR. So long as $B$ is not the MBR, it too has room to grow. If $A$ overlap MBR, then so long as $A$ partially intersects $B$, then overlap is possible.

www.josis.org 
Rule 4.7. A relation cannot be disjoint if (1) the opposite edge neighbour to an overlap is not disjoint and both of the remaining edge neighbors are meet or disjoint (Figure 11a), (2) a corner tile not edge-adjacent to a non-empty intersection has relation meet and the MBR and at least one other tile is non-empty (Figure 11b), or (3) there are tiles with relation meet on opposite sides of the $M B R$ with no additional non-empty intersections (Figure 11c).

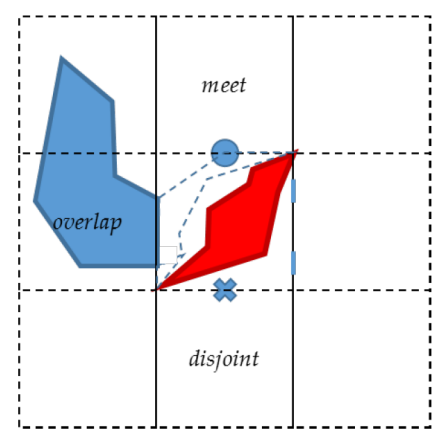

(a)

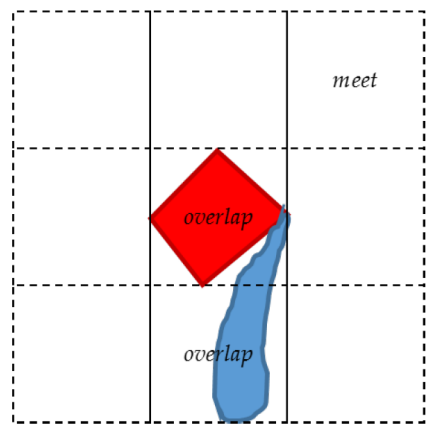

(b)

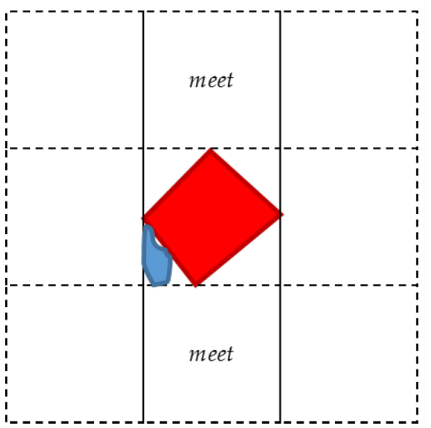

(c)

Figure 11: Three possible cases from Rule 4.7: (a) Rule 4.7.1, (b) Rule 4.7.2, and (c) Rule 4.7.3. In (a), the object presents a barrier to attaining the slots necessary on both sides of the MBR. In (b), the object again creates a barrier to reaching the opposite side of the MBR as it cannot enter the two tiles that share an edge with it. Finally, in (c), the object again precludes the crossing as the object is not allowed to leave the tile in this case in any direction.

Proof. As shown in Figure 11, all three of these scenarios present an instance where the object must cross the MBR without leaving it. As soon as the object reaches the MBR, it must intersect $B^{\prime}$ s boundary to continue as it is not allowed to leave to go around it as it has disjoint or meet as its neighbour (Figure 9).

Rule 4.8. A relation cannot be meet if the opposite edge neighbor to an overlap or a meet is not disjoint and both of the remaining edge neighbors are meet or disjoint and both corner tiles adjacent to the opposite edge neighbor are not meet (Figure 11a), or all four corners are meet while both edge neighbors are strictly disjoint (Figure 9).

Proof. The rule has two parts. The first is equivalent to Rule 4.7, just applied to meet instead (Figure 11). If the object were allowed to attain the corner points as its edge intersections, then the relation meet is possible. The second part of the rule prohibits the corner tile from being the intersection, as that common boundary point is shared amongst the four adjacent tiles, precluding disjoint in any of them.

Rule 4.9. If the relation between the object and the $M B R$ is disjoint, then the relation disjoint is the only possible relation between the two objects.

Proof. By composition, disjoint $;($ covers or equal $)=$ disjoint . 
Rule 4.10. If the relation between the object and the $M B R$ is meet, the relations meet and disjoint must be possible.

Proof. By composition, meet $;($ covers or equal $)=($ disjoint or meet $)$.

\section{Analysis of topological augmentation rules applied to the topological determination problem}

The rules derived in Section 4 provide a mechanism by which to further understand the possibilities from within each of the congruence classes. In this section, the rules from Section 4 are applied in two manners: (1) can the relation be exhibited between $A$ and $B$ in that class (which is already known from Section 3), and (2) can the relation be identified between $A$ and $B$, independent of the $A$ and $B$ defining the topologically augmented direction relation matrix symbol? Both tables will consider each specific topological augmentation that can apply to a direction relation matrix symbol as an individual entity.

\subsection{Symbols that have a topological relation as a consequence}

Figure 13 dictates the mapping of topological relations identified in Section 3, but gives a number of topological augmentations for which that symbol may occur. The numbers presented in Figure 13 are derived explicitly from the rules in Section 4. To demonstrate the process, consider the topological augmentation presented in Figure 12. To summarize, the following are the applications of the rules to Figure 12.

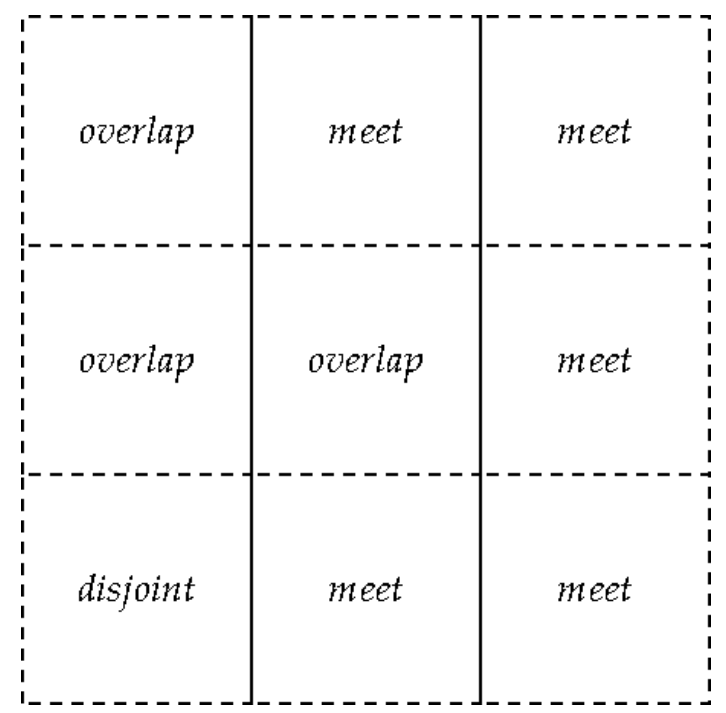

Figure 12: Test case to demonstrate the application of Rules 4.1-4.10.

- Rule 4.1 does not apply as the MBR does not have relation coveredBy or equal, nor is the relation in $W_{B}$ meet, therefore the topological relation cannot be equal.

www.josis.org 
- Rule 4.2 does not apply as the MBR does not have relation inside, nor is the relation for any of $N_{B}, S_{B}, E_{B}$, and $W_{B}$ meet, therefore the topological relation cannot be inside.

- Rule 4.3 similarly does not apply as the MBR does not have relation coveredBy or inside, nor is the relation for all of $N_{B}, S_{B}, E_{B}$, and $W_{B}$ meet or disjoint, therefore the topological relation cannot be coveredBy.

- Rule 4.4 does not apply as there exists at least one relation in $N_{B}, S_{B}, E_{B}$, and $W_{B}$ that is not overlap, therefore the topological relation cannot be contains.

- Rule 4.5 applies as none of $N_{B}, S_{B}, E_{B}$, and $W_{B}$ have relation disjoint, therefore the topological relation can be covers.

- Rule 4.6 applies as the MBR has relation overlap, therefore the topological relation can be overlap.

- Rule 4.7 applies, as the object is sandwiched between $N_{B}$ and $S_{B}$ to reach $E_{B}$ from $W_{B}$, therefore the topological relation cannot be disjoint.

- Rule 4.8 does not apply as the two corner tiles $N E_{B}$ and $S E_{B}$ are meet, therefore meet is a possibility as the topological relation.

- Rule 4.9 does not apply as the MBR does not have relation disjoint.

- Rule 4.10 does not apply as the MBR does not have relation meet.

From this inventory, the relations meet, overlap, and covers can be attained from this topological augmentation of a congruence class 8 direction relation matrix. This reduces the total by one for possible relations, eliminating disjoint.

\subsection{Identifying the topological relation from a particular topologically augmented direction relation matrix symbol}

While Figure 13 always demonstrates a reduction from Guo and Du [23], it does not answer the question as to whether or not topological augmentation can determine the actual relation between two regions $A$ and $B$. To do that, Figure 14 is presented to mirror Figure 13 , determining how many relations are uniquely identified by a topological augmentation from that congruence class. Like in Figure 13, Figure 14 treats each class as if it were represented by a single symbol.

\subsection{Analysis of the tables}

Figures 12 and 13 have detailed how the relations are distributed amongst individual congruence classes of the direction relation matrix when topological augmentation is applied. Now the following questions can be addressed:

- What percentage of topological augmentations reduce the overall topological diversity exhibited within a particular direction relation matrix symbol?

- What percentage of topological augmentations allow each of the particular topological relations?

- What percentage of topological augmentations uniquely identify a single topological relation?

- What percentage of topological augmentations identify each of the particular topological relations? 


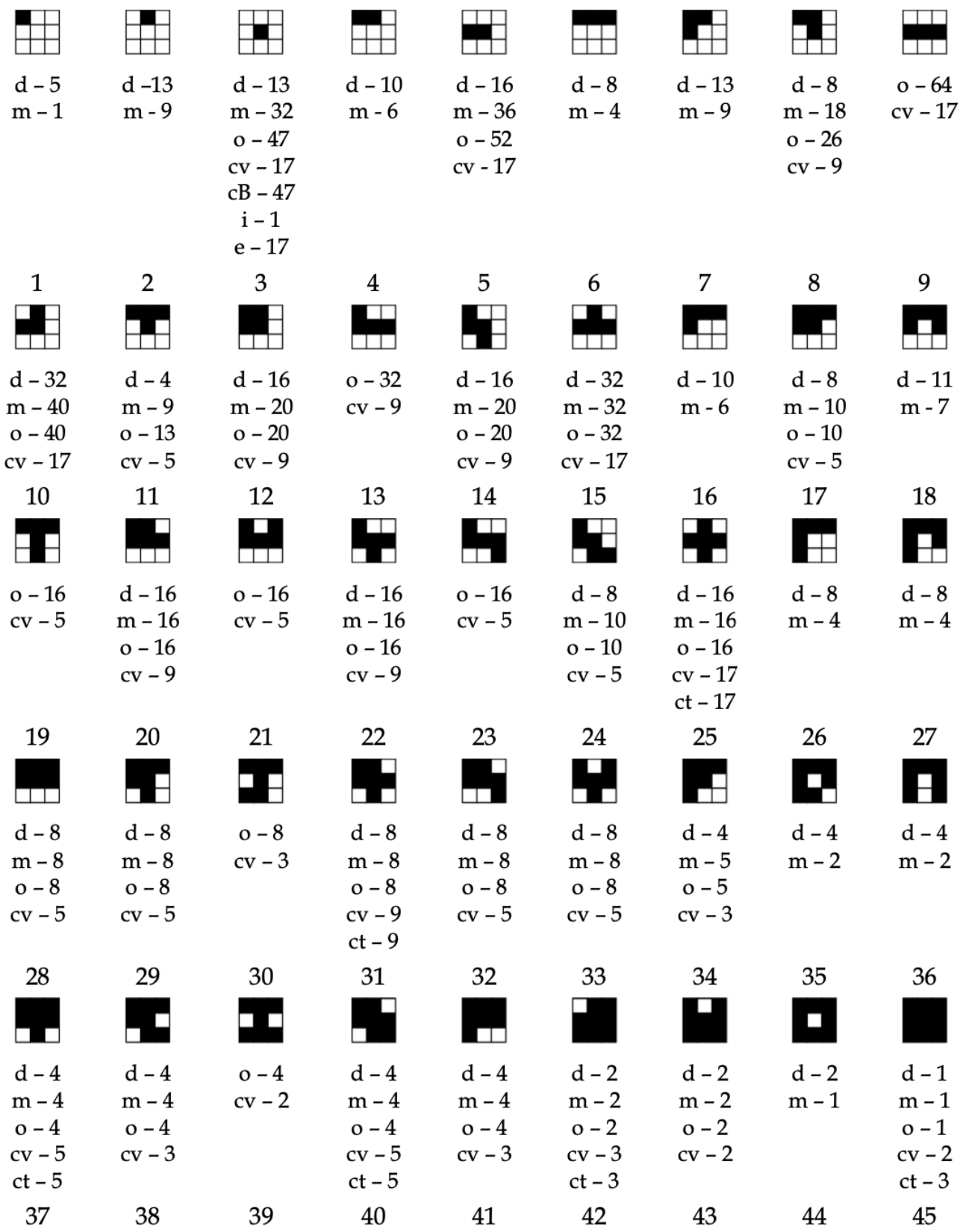

Figure 13: Topological augmentations from the congruence class that can produce the named topological relation. The numbers presented reflect only one symbol from the congruence class. 


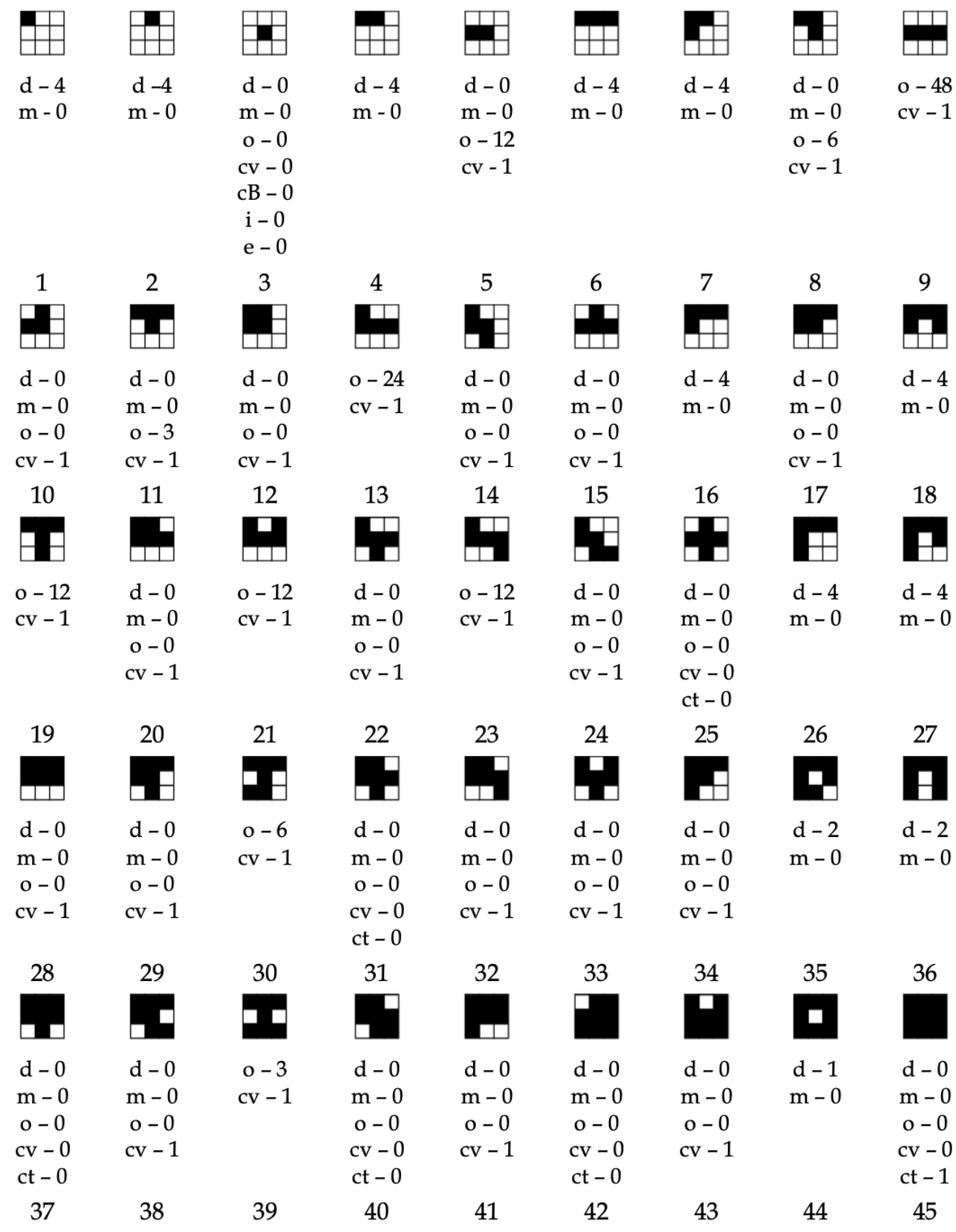

Figure 14: Topological augmentations from the congruence class that always produce the named topological relation. The numbers presented reflect only one symbol from the congruence class. 


\subsubsection{Topological reduction}

To answer the question of topological reduction, we first must scale up the numbers in Figure 12 by the number of symbols in the particular congruence class. This number can be found in previous work [6]. We can then consider the reduction number to be all topologically augmented symbols which do not contain all of the symbols reflected in the congruence class. The resultant table can be found in previous work [6]. As a function of these appropriate scalings, 2,128 of the 3,084 topological augmentations $(69 \%)$ reduce the number of topological relations as prescribed by Guo and Du (with the addition of Theorem 3.1).

\subsubsection{Prevalence of topological relations}

To answer the question of topological prevalence, we again can refer to the same tables as suggested in Section 5.3.1. Instead of being concerned with reduction, we are solely concerned with the presence of a particular topological relation within the table. The results are as follows, in order of topological prevalence:

- overlap - 2,432 occurrences $(78.9 \%)$

- meet $-1,906$ occurrences $(61.8 \%)$

- disjoint - 1,808 occurrences $(58.6 \%)$

- covers - 1,104 occurrences (35.8\%)

- contains - 98 occurrences $(3.2 \%)$

- coveredBy -47 occurrences $(1.5 \%)$

- equal - 17 occurrences $(0.6 \%)$

- inside - 1 occurrence $(0.1 \%)$

This list has a few interesting qualities. Certain relations are incredibly specific, such as inside and equal, while others such as overlap, meet, disjoint, and covers are highly available. With respect to neighbourhood graphs, this list is not symmetrical, however, in all cases boundary contact is more prevalent than not when considering its corresponding RCC-5 abstraction [3].

\subsubsection{Topological identification}

Using a similar technique, we can also consider the percentage of topological augmentations that identify a particular topological relation unambiguously. These topological augmentations represent the ultimate prize for direction and topological reasoning: the exchange of information across models for free. Unlike reduction, however, we need not consider what is paired with what, as identification already suggests decoupling. By using the same scale factor for symbol cardinality, we can determine that 882 topological augmentations $(28.6 \%)$ identify a particular topological relation. While this is roughly two of every seven, this is a substantial increase from Guo and Du. 39 of the 45 congruence classes contain at least one topological augmentation that will identify a topological relation.

\subsubsection{Prevalence of identification}

Following the same course as Section 5.3.2, we now consider the amount of times a particular topological relation is identified by a topological augmentation. The list is as follows:

WwW.josis.org 
- overlap - 594 occurrences (19.3\% overall, $67.3 \%$ of all identifiers, $24.4 \%$ of all overlap occurrences)

- disjoint - 209 occurrences (6.8\% overall, $23.7 \%$ of all identifiers, $11.6 \%$ of all disjoint occurrences)

- covers - 144 occurrences (4.7\% overall, $16.3 \%$ of all identifiers, $13.0 \%$ of all covers occurrences)

- contains - 1 occurrence $(0.1 \%$ overall, $0.1 \%$ of all identifiers, $1 \%$ of all contains occurrences)

While we saw before that particular relations were incredibly specific, that specificity is not enough to identify the relations. Curiously, covers can be identified, but not meet or coveredBy. Similarly, inside cannot be identified, however disjoint and contains can. Unsurprisingly, most occurrences of any topological relation are not identifiable, with no single topological relation surpassing the $28.6 \%$ overall rate.

\section{Conclusions and future work}

Guo and Du [23] studied the interplay of the direction relation matrix [21] and topological relations [17] to identify whether or not the direction relation matrix could stand in some cases as a surrogate for topology, or the degree to which the direction relation matrix could effectively limit the topological variability present, similar to the effect of compositions on the universal relation [13]. They found that there were no instances where a direction relation matrix could stand for a topological relation, though in all cases, it was better than nothing insofar as the universal relation was never encountered. Guo and Du's work missed a fundamental rule (as added in Section 3), but even if enforced, would not lead to topological determination, thus not negating their claim.

In this paper, we applied topological augmentation [8] to this problem space, hoping to find topological augmentations that would further reduce the results of Guo and Du [23] to a more limited set of topological relations, or perhaps in an ideal world lead to an exact topological relation. It was demonstrated that in $69 \%$ of topological augmentations, the results from Guo and $\mathrm{Du}$ for their corresponding direction relation matrix symbol was reduced by at least one topological relation, a sizable gain. The research also demonstrated that for some $28.6 \%$ of topological augmentations, a precise topological relation could in fact be determined, including results from 39 different congruence classes representing 202 of the 218 realizable direction relation matrix symbols. While certain classes fare better than others-both in reduction and determination-there is an importance to this connection between direction and topology. When topology fails human beings in our decision making processes, we often move toward directions to catch us. This can be seen in the list of prepositions in the English language: the two dominant areas in the classification of prepositions that are spatial in nature are topological and directional as opposed to distance [27]. Any instance where topological reasoning can be additionally inferred creates stronger reasoning systems on a spatial level.

Topological augmentation is but a fledgling technique, meaning that there are numerous ways to expand this work. Such areas include:

- Using topological augmentation for purposes that are not based on simple regions, such as for line-region relations [33], 
- Using topological augmentation in spherical embedding spaces, opening up additional possibilities [14], and

- Developing a GIS implementation of the theory by exploiting known pieces of GIS, including the MBR function and the topological query operators [5].

\section{Acknowledgments}

The author wishes to acknowledge support from National Science Foundation grant 2019470 (PIs McGill, Gotelli, Waring, Niles, Dube) for partial support of this work.

\section{References}

[1] Adams, C., And Franzosa, R. Introduction to Topology: Pure and Applied. Pearson Prentice-Hall, Upper Saddle River, NJ, 2008.

[2] BARRETT, J. Identifying viable symbols in 3d qualitative direction partitions. Tech. Rep. TR 1, Upward Bound Math-Science, University of Maine, July 2013.

[3] BennetT, B. Spatial reasoning with propositional logics. In Principles of Knowledge Representation and Reasoning (1994), pp. 51-62.

[4] Chen, T., Schneider, M., Viswanathan, G., And Yuan, W. The objects interaction matrix for modelling cardinal directions in spatial databases. In International Conference on Database Systems for Advanced Applications (Tsukuba, Japan) (2010), pp. 218232.

[5] Clementini, E., Sharma, J., And Egenhofer, M. Modelling topological spatial relations: Strategies for query processing. Computers and Graphics 6, 18 (1994), 815822.

[6] Dube, M. Algebraic Refinements of Direction Relations through Topological Augmentation. PhD thesis, University of Maine, 2016.

[7] DubE, M. Beyond homeomorphic deformations: Neighborhoods of topological changes. In Advancing Geographic Information Science: The Past and Next Twenty Years, H. Onsrud and W. Kuhn, Eds. GSDI Press, 2016, ch. 10, pp. 137-152.

[8] DubE, M. Topological augmentation: A step forward for qualitative partition reasoning. Journal of Spatial Information Science 1, 14 (2017), 1-29.

[9] Dube, M., AND Egenhofer, M. An ordering of convex topological relations. In International Conference on Geographic Information Science (Columbus, OH) (2012), pp. 7286.

[10] Dube, M., And Egenhofer, M. Binary topological relations on the digital sphere. International Journal of Approximate Reasoning 1, 116 (2020), 62-84.

[11] Dube, M., Egenhofer, M., BArrett, J., And Simpson, N. Beyond the digital jordan curve: Unconstrained simple pixel-based raster relations. International Journal of Approximate Reasoning 1, 54 (2019), 100906.

www.josis.org 
[12] Dube, M., Egenhofer, M., Lewis, J., Stephen, S., And Plummer, M. Swiss canton regions: A model for complex objects in geographic partitions. In International Conference on Spatial Information Theory (Santa Fe, NM) (2015), pp. 309-330.

[13] Egenhofer, M. Deriving the composition of binary topological relations. Journal of Visual Languages and Computing 2, 5 (1994), 133-149.

[14] Egenhofer, M. Spherical topological relations. Journal on Data Semantics III 1, 1 (2005), 25-49.

[15] Egenhofer, M. A reference system for topological relations between compound spatial objects. In International Conference on Conceptual Modelling (Gramado, Brazil) (2009), pp. 307-316.

[16] Egenhofer, M., AND Franzosa, R. Point-set topological spatial relations. International Journal of Geographical Information Science 2, 5 (1991), 161-174.

[17] Egenhofer, M., ANd Herring, J. Categorizing binary topological relations between regions, lines, and points in geographic databases. Tech. Rep. TR 1, National Center for Geographic Information and Analysis, July 1990.

[18] Egenhofer, M., AND MARK, D. NaÃŕrve geography. In International Conference on Spatial Information Theory (Semmering, Austria) (1995), pp. 1-15.

[19] Egenhofer, M., And Sharma, J. Topological relations between regions in $R^{2}$ and $Z^{2}$. In International Symposium on Spatial Databases (Singapore) (1993), pp. 316-336.

[20] FRANK, A. Qualitative spatial reasoning about distances and directions in geographic space. Journal of Visual Languages and Computing 4, 3 (1992), 343-371.

[21] Goyal, R., AND Egenhofer, M. Cardinal directions between extended spatial objects. IEEE Transactions on Knowledge and Data Engineering 1, 21 (2000), 22-31.

[22] Guerra-Filho, G., Medeiros, C., And De Rezende, P. A formalization of topological relations between simple spatial objects. In Advances in Spatial Data Handling (Bonn, Germany) (2013), pp. 61-75.

[23] GUO, L., AND DU, S. Deriving topological relations between regions from direction relations. Journal of Visual Languages and Computing 6, 20 (2009), 368-384.

[24] Kor, A., AND BenNetT, B. A hybrid reasoning model for whole and part cardinal direction relations. Advances in Artificial Intelligence 1, 3 (2009), 3.

[25] KuIPERS, B. Modeling spatial knowledge. Cognitive Science 2, 2 (1978), 129-153.

[26] KuRATA, Y. The 9+-intersection: A universal framework for modeling topological relations. In International Conference on Geographic Information Science (Park City, UT) (2008), pp. 181-198.

[27] LANDAU, B., AND JACKENDOFF, R. What and where in spatial language and spatial cognition. Behavioral and Brain Sciences 2, 16 (1993), 217-238. 
[28] Lewis, J., Dube, M., And Egenhofer, M. The topology of spatial scenes in $R^{2}$. In International Conference on Spatial Information Theory (Scarborough, UK) (2013), pp. 495515.

[29] Lewis, J., AND EgENHOfER, M. Oriented regions for linearly conceptualized features. In International Conference on Geographic Information Science (Vienna, Austria) (2014), pp. 333-348.

[30] LI, S. A complete classification of topological relations using the 9-intersection method. International Journal of Geographical Information Science 6, 20 (2006), 589-610.

[31] LI, S., AND YING, M. Region connection calculus: Its models and composition table. Artificial Intelligence 1-2, 145 (2003), 121-146.

[32] LIU, Y., WANG, X., JIN, X., AND WU, L. On internal cardinal direction relations. In International Conference on Spatial Information Theory (Ellicottville, NY) (2005), pp. 283299.

[33] Mark, D., AND Egenhofer, M. Modeling spatial relations between lines and regions: Combining formal mathematical models and human subjects testing. Cartography and Geographic Information Systems 4, 21 (1994), 195-212.

[34] Millner, R. Bigraphs and their algebra. Electronic Notes in Theoretical Computer Science 1, 209 (2008), 5-19.

[35] Mitra, D. Qualitative reasoning with arbitrary angular directions. In Proceedings of the AAAI-02 W20 Workshop on Spatial and Temporal Reasoning (Edmonton, Alberta) (2002), pp. 1-6.

[36] MoRATZ, R. Representing relative direction as a binary relation of oriented points. In European Conference on Artificial Intelligence (Riva del Garda, Italy) (2006), pp. 407-411.

[37] Papadias, D., Sellis, T., Theodoridis, Y., And Egenhofer, M. Topological relations in the world of minimum bounding rectangles: A study with r-trees. ACM SIGMOD 2, 24 (2008), 92-103.

[38] Peuquet, D., And Ci-XIAng, Z. An algorithm to determine the directional relationship between arbitrarily-shaped polygons in the plane. ACM SIGMOD 1, 20 (1987), 65-74.

[39] RANDELL, D., CUI, Z., AND COHN, A. A spatial logic based on regions and connection. In Proceedings of the 3rd International Conference on Principles of Knowledge Representation and Reasoning (Cambridge, MA) (1992), pp. 165-176.

[40] Reis, R., Egenhofer, M., AND Matos, J. Conceptual neighborhoods of topological relations between lines. In The 13th International Symposium on Spatial Data Handling (Montpelier, France) (2008), pp. 557-574.

[41] SCHNEIDER, M., AND BEHR, T. Topological relationships between complex spatial objects. ACM Transactions on Database Systems 1, 31 (2006), 39-81.

[42] Sharma, J. Integrated Spatial Reasoning in Geographic Information Systems: Combining Topology and Direction. PhD thesis, University of Maine, 1996.

www.josis.org 
[43] SHEN, J., ZHANG, L., AND CHEN, M. Topological relations between spherical spatial regions with holes. International Journal of Digital Earth 1, 4 (2018), 1-28.

[44] Shen, J., Zhou, T., AND Chen, M. A 27-intersection model for representing detailed topological relations between spatial objects in two-dimensional space. ISPRS International Journal of Geo-Information 2, 6 (2017), 37.

[45] TALmY, L. How language structures space. In Spatial Orientation: Theory, Research, and Application, H. Pick and L. Acredolo, Eds. Springer, 1983, ch. 11, pp. 225-282.

[46] Worboys, M. Using maptrees to characterize topological change. In International Conference on Spatial Information Theory (Scarborough, UK) (2013), pp. 74-90.

[47] ZimmermanN, S. Measuring without measures: The delta-calculus. In International Conference on Spatial Information Theory (Semmering, Austria) (1995), pp. 59-67. 